\title{
O uso do disclosure como ferramenta de gestão da qualidade e segurança do
}

\section{paciente: uma revisão sistemática}

\author{
The use of disclosure as a tool for quality management and patient safety: a systematic review \\ O el uso de la divulgación como herramienta para la gestión de la calidad y la seguridad del
}

paciente: una revisión sistemática

Recebido: 18/05/2021 | Revisado: 29/05/2021 | Aceito: 03/10/2021 | Publicado: 04/10/2021

\author{
Elaine Rossi Ribeiro \\ ORCID: https://orcid.org/0000-0003-3492-217X \\ Faculdades Pequeno Príncipe, Brasil \\ E-mail: elaine.rossi@hotmail.com \\ Cássia Laura Gheller Bertoldo \\ ORCID: https://orcid.org/0000-0002-4417-4622 \\ Faculdades Pequeno Príncipe, Brasil \\ E-mail: cassia.gheller@gmail.com \\ Ana Clara Kunz \\ ORCID: https://orcid.org/0000-0002-5194-1340 \\ Faculdades Pequeno Príncipe, Brasil \\ E-mail: anaaclarakunz@gmail.com
}

\begin{abstract}
Resumo
Introdução: todos os anos, milhares de eventos adversos ocorrem na assistencia á saúde, sendo que $2 / 3$ destes incidentes são causados por erro humano. Apesar da obrigação ética, tais erros não são comumente divulgados e a prática da comunicação efetiva com pacientes e familiares em grande medida é negligenciada. Objetivo: analisar o uso do disclosure, como ferramenta de gestão da qualidade e segurança, entre profissionais de saúde e pacientes. Método: o método utilizado foi revisão sistemática, submetida e publicada no prospero, utilizando-se os bancos de dados pubmed, scielo, lilacs e bvs. As palavras-chave para realização da busca foram "disclosure", "medical errors" e "patient safety". Resultados: foram analisados 13 artigos sendo possível evidenciar pontualmente as categorias denominadas: erros médicos e segurança do paciente, processos e protocolos e estado da arte. Conclusão: o processo de disclosure não recebe atenção suficiente na comunidade científica ou em instituições de saúde do mundo, considerando-se que poucos países apresentam práticas formalizadas e institucionalizadas por meio de protocolos ou guidelines sobre essa técnica de comunicação efetiva primordialmente humana.
\end{abstract}

Palavras-chave: Disclosure; Erros médicos; Segurança do paciente.

\begin{abstract}
Intro: Every year, thousands of adverse events occur in health care, and $2 / 3$ of these incidents are caused by human error. Despite the ethical obligation, such errors are not commonly disclosed and the practice of effective communication with patients and families is largely neglected. Objective: to analyze the use of disclosure, as a tool for quality and safety management, among health professionals and patients. Method: The method used was a systematic review, submitted and published in the Prospero, using the databases pubmed, scielo, lilacs and bvs. The keywords used for the search were "disclosure", "medical errors" and "patient safety". Results: 13 articles were analyzed and it was possible to evidence punctually the categories named: medical errors and patient safety, processes and protocols and state of the art. Conclusion: the disclosure process does not receive enough attention in the scientific community or in health institutions around the world, considering that few countries have formalized and institutionalized practices through protocols or guidelines on this technique of effective communication primarily human.
\end{abstract}

Keywords: Disclosure; Medical errors; Patient safety.

\section{Resumen}

Introducción: cada año se producen miles de eventos adversos en la asistencia sanitaria, y dos tercios de estos incidentes están causados por errores humanos. A pesar de la obligación ética, estos errores no se suelen revelar y se descuida en gran medida la práctica de una comunicación eficaz con los pacientes y las familias. Objetivo: analizar el uso de la divulgación, como herramienta de gestión de la calidad y la seguridad, entre los profesionales sanitarios y los pacientes. Método: el método utilizado fue una revisión sistemática, presentada y publicada en el Prospero, utilizando las bases de datos pubmed, scielo, lilacs y bvs. Las palabras clave utilizadas en la búsqueda fueron "disclosure", "medical errors" y "patient safety". Resultados: se han analizado 13 artículos y se han puesto de 
manifiesto las categorías denominadas: errores médicos y seguridad del paciente, procesos y protocolos y estado del arte. Conclusión: el proceso de divulgación no recibe suficiente atención en la comunidad científica o en las instituciones de salud del mundo, considerando que pocos países presentan prácticas formalizadas e institucionalizadas por medio de protocolos o directrices sobre esta técnica de comunicación eficaz primordialmente humana.

Palabras clave: Divulgación; Errores médicos; Seguridad del paciente.

\section{Introdução}

A segurança do paciente somente começou a receber a atenção devida em 1999, mesmo sabendo-se que Hipoócrates, com sua célebre frase primum non nocere (em primeiro lugar não causar danos) já sinalizava para esta preocupação. (Motta filho et al, 2013). O relatório “To Err Is Human: Building a Safer Health System”, que chamou a atenção do público ao problema das mortes de pacientes devido a erros médicos, aprofundou tais preocupações até que em 2004, foi lançada a Aliança Mundial para a Segurança do Paciente, pela Organização Mundial da Saúde (OMS) (Motta Filho et al, 2013), e no Brasil, o Ministério da Saúde instituiu o Programa Nacional de Segurança do Paciente (PNSP), por meio da Portaria MS/GM $\mathrm{n}^{\circ} 529$, de $1^{\circ}$ de abril de 2013 (Brasil, 2004).

Segundo a Organização Mundial de Saúde (OMS), segurança do paciente é definida como cuidados com o paciente visando reduzir erros e minimizar efeitos adversos (Kisuule et al, 2015). A Classificação Internacional de Segurança do Paciente, desenvolvida pela OMS tem como objetivo compartilhar informações e cessar dúvidas sobre segurança do paciente em um aspecto mundial. O objetivo principal da Classificação para Segurança do Paciente é a criação de uma linguagem global comum em relação a segurança do paciente, para que instituições de todo o mundo possam classificação os eventos de forma similar, compartilhando experiências.

A prevalência de erros médicos tem sido uma área de grande interesse ao longo das duas últimas décadas. As altas taxas de eventos adversos (EAs), em sua grande maioria, evitáveis, são uma indicação de que erros estão ocorrendo. Esses erros são diversos, comuns e ocorrem em todos os níveis do sistema de saúde. Além disso, a magnitude e o potencial dos erros médicos são enormes e se tornando um elemento fixo na área de saúde. Erros médicos e EAs são os principais motivos dos danos causados aos pacientes, com a gravidade variando de pouca ou nenhuma consequência a erros fatais. (Kalra et al, 2013).

Existem muitos motivos pelos quais ocorrem os erros médicos. A saúde é um ambiente complexo devido a processos de doença multifacetados, também como equipe médica, equipamentos, infraestrutura, políticas organizacionais, e procedimentos. Deve-se perceber que certo grau de erro é inevitável dentro de cada tarefa humana e, portanto, algum grau deve ser aceito. A maioria dos erros é devido a falhas do sistema de saúde, não apenas dos indivíduos. Soluções eficazes não surgirão se apenas os indivíduos forem alvos do problema. Na verdade, a cultura de culpa é um fator importante para o número elevado inaceitável de erros médicos. (Kalra et al, 2013)

Neste sentido, para fortalecer a cultura de segurança, o Instituto Brasileiro de Segurança do Paciente -IBSP - diz em artigo denominado "Disclosure precisa integrar a cultura de segurança do paciente", que: o processo estruturado de comunicação com o paciente e seus familiares quando existe um dano não intencional grave, ou potencialmente grave, durante o processo da assistência é conhecido pelos profissionais de saúde como disclosure. (IBSP, 2015)

O disclosure é umas das conversas mais complexas e difíceis na saúde, um verdadeiro desafio para médicos, especialmente em algumas áreas da medicina a qual o contato com os pacientes é limitado (Mclennan et al, 2015). Um estudo conduzido por Gallagher et al (2007) revela que apenas 30\% dos médicos que vivenciaram um evento adverso grave em sua prática havia realizado disclosure e se desculpado pela falha, apontando para algumas dificuldades, como o medo do processo, o desconforto em enfrentar o paciente e a falta de conhecimento e habilidade para a comunicação adequada.

Para ter uma comunicação efetiva algumas estratégias são essenciais como por exemplo: terminologias que sejam entendidas pelo paciente e familiares, uso da empatia, tempo adequado de conversa, certificar que a informação foi entendida e 
ser compreensivo em relação à linguagem e cultura do paciente. (Canadian Disclosure Guidelines, 2011)

Ao desenvolver e implementar uma política ou processo de disclosure, deve-se entender que cada paciente e cada incidente de segurança do paciente é único. O processo de divulgação requer flexibilidade para garantir que seja afetivo e atenda às necessidades de informação de cada paciente individualmente (Canadian Disclosure Guidelines, 2011)

Diante deste cenário, ainda em construção, questiona-se: O disclosure tem sido usado pelos profissionais de saúde, como ferramenta de gestão da qualidade e segurança do paciente? Sendo assim, determinou-se como objetivo deste estudo, analisar o uso do disclosure, como ferramenta de gestão da qualidade e segurança, realizado entre profissionais de saúde e pacientes/familiares.

\section{Metodologia}

O tipo de estudo desenvolvido foi uma revisão sistemática, sendo que esta metodologia propõe identificar estudos sobre um tema em questão, aplicando métodos explícitos e sistematizados de busca; e avaliar a qualidade e validade desses estudos, assim como sua aplicabilidade no contexto em que as mudanças serão implementadas (Sampaio et al, 2007).

Essa revisão sistemática foi submetida e publicada no PROSPERO, com o seguinte código de identificação CRD 160317.

Para isso foram utilizados os bancos de dados PubMed, Scielo, Lilacs e BVS. As palavras-chave para realização da busca foram "Disclosure", "Medical errors" e "Patient safety", com o operador Booleano AND. Tais descritores foram escolhidos mediante pergunta de pesquisa que utilizou o acrônimo PVO, em que a população representa os profissionais da área da saúde, pacientes e familiares; a variável, o uso do disclosure como ferramenta de gestão da qualidade e segurança do paciente; e o outcome ou resultados, a utilização do disclosure. Os critérios de inclusão foram: artigos do ano de 2010 até 2020, em inglês, português, espanhol, e de acesso livre.

Em continuidade, os pesquisadores analisaram os 27 itens do PRISMA (Preferred Reporting Items for Systematic Reviews and Meta-Analyses: The PRISMA Statement), para conferência das partes integrantes essenciais de uma revisão sistemática, segundo Moher et al. (2009).

\section{Resultados e Discussão}

Na busca, foram encontrados 228 artigos na PubMed, 502 artigos na BVS e 2 artigos na LILACS. Foram selecionados 44 artigos pela leitura inicial de título e resumo, momento em que 20 foram excluídos por não responderem a pergunta de pesquisa. Sendo assim, 24 artigos foram lidos na integra, de forma independente pelas pesquisadoras e para atender o objetivo da pesquisa, foram selecionados para amostra final, 13 artigos. O processo está ilustrado no Fluxograma 1 a seguir: 
Fluxograma 1. Processo de seleção de artigos.

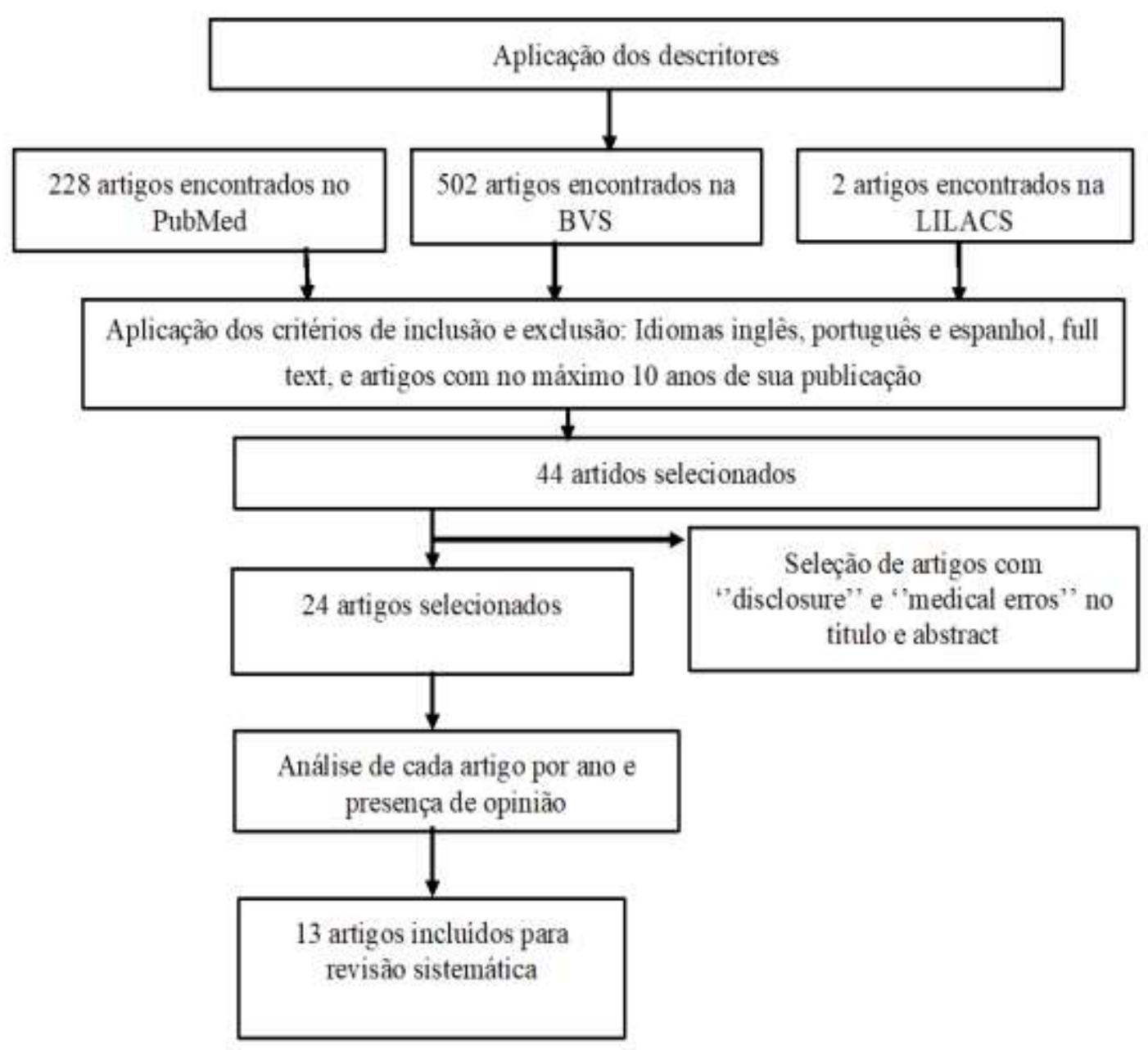

Fonte: Autores (2020).

Os treze artigos selecionados foram publicados entre o período de 2010 a 2020. Desses, seis foram produzidos no continente americano, quatro no europeu e três no asiático. O ano que apresentou mais publicações foi o de 2016 , com três artigos. Esse é seguido pelos anos 2011, 2012, 2014 e 2015, com dois artigos. Os anos de 2010 e 2013 somente tiveram uma publicação.

Para facilitar a compreensão dos resultados, o conteúdo foi subdividido em três categorias, de forma didática. Após leituras aprofundadas dos 13 artigos que compuseram a amostra final, foi possível evidenciar que os temas tratados, embora discutam o disclosure, apontavam, em conjunto, para os assuntos demonstrados a seguir:

3.1 - Erros Médicos e Segurança do paciente

3.2 - Processos e protocolos

3.3 - Estado da arte

\subsection{Erros médicos e Segurança do paciente}

43 milhões de eventos adversos ocorrem na medicina todos os anos. Sendo que, 2/3 desses incidentes são causados por um erro humano. Apesar das obrigações éticas, os erros são divulgados em menos de 1/3 de todos os casos, destes, apenas metade explica o que aconteceu, 1/3 pede desculpa e muito poucos discutem modos de prevenção de futuras recorrências. 
(Hannawa et al, 2016)

De acordo com Mclennan et al (2015), a anestesiologia foi considerada a especialidade médica que primeiro discutiu sobre os erros médicos. Em pesquisa realizada com 542 anestesiologistas ativos clinicamente na Suíça, foi analisado que $98 \%$ deles relataram já estarem envolvidos em erros de segurança do paciente. $78 \%$ concordaram que erros médicos são problemas importantes e sérios na área da saúde, e a maioria concordou que a comunicação do erro é importante para a segurança do paciente. Todavia, de todos os anestesiologistas, apenas $34 \%$ já realizou disclosure de um erro grave, enquanto $75 \%$ relataram ter feito o disclosure de um pequeno erro, mostrando que existem barreiras para a comunicação de intercorrências.

Em relação à segurança do paciente, Daud-Gallotti et al (2011) apontam que é necessário prestação de cuidados centrados no paciente; comunicação com os pacientes e familiares; consciência da ocorrência, prevenção, gerenciamento e disclosure; segurança entre equipes; defesa da ética médica; práticas baseadas em evidências; e conhecimento em tecnologia da informação e em estratégias. Outro fator abordado pelo autor, é a educação médica que carece de treinamentos, resultando em estudantes não preparados para cumprir com as responsabilidades que cercam o cuidado.

Em estudo desenvolvido pelo autor, na Faculdade de Medicina da Universidade de São Paulo, 95 estudantes de medicina do quinto ano foram avaliados através de estações com pacientes padronizados. A média geral dos exames clínicos foi de 85,89 , sendo que a média isolada da estação "segurança do paciente" ficou em 87,59. Além disso, os alunos foram capazes de explicar o erro médico e as suas consequências, obtendo média de 94,77 nesse quesito. (Daud-Gallotti et al, 2011)

$\mathrm{O}$ autor aponta que as médias altas obtidas pelos estudantes se devem a incorporação de um programa educacional de segurança do paciente introduzido pela faculdade em 2007. Os alunos assistem a duas palestras por rotação durante o quinto ano, que abordam temas como definição de erro médico; epidemiologia e disclosure; quase acidentes; eventos adversos; assistência centrada no paciente; relação médico-paciente; e ética. Ao fim do experimento, os alunos puderam avaliar a instituição desse programa de segurança. 94\% dos 95 participantes reconheceram a importância de aprender, discutir, avaliar e receber feedback sobre o aprendizado do disclosure durante o estágio. (Daud-Gallotti et al, 2011)

Nesta mesma linha, o estudo aplicado em 191 estudantes na Alemanha, com o intuito de avaliar a implementação curricular de segurança do paciente, evidenciou-se que há uma falta de abordagem a "erros médicos" durante a graduação. $64 \%$ dos alunos apontaram que gostariam de receber treinamento nessa área. Sendo que 1/4 dos participantes admitiram já ter cometido algum erro durante a formação. (Kiesewetter et al, 2014)

Em relação à segurança no ambiente cirúrgico, o Brasil aderiu à campanha Cirurgias Seguras Salvam Vidas, em 2008. Esse movimento tem como objetivo a utilização de uma lista de verificação aplicada em três diferentes momentos, em todas as cirurgias: antes da anestesia (Sign In), antes da incisão (Time Out) e antes da retirada do paciente da sala de cirurgia (Sing Out). (Motta Filho et al 2013). Levando em consideração esse protocolo, realizou-se uma pesquisa durante o $44^{\circ}$ Congresso Brasileiro de Ortopedia e Traumatologia, em 2012, em que 502 profissionais responderam a um questionário sobre cirurgia segura. Destes, 199 (39,6\%) alegaram ter vivenciado algum erro nos últimos seis meses. Quando questionados sobre o protocolo instituído pela OMS, 65,3\% afirmaram o desconhecer total ou parcialmente e 72,1\% relataram não terem sido treinados para utilizar a lista de verificação. (Motta Filho et al 2013)

\subsection{Processos e protocolos}

O DA\&O, sigla para Disclosure, Apology e Offer, é a prática que enfoca a comunicação com pacientes e familiares, além de uma abordagem aos erros. Esse é baseado em princípios, como identificar de forma proativa os eventos adversos distinguir entre as lesões causadas por doenças negligenciadas e as decorrentes de complicações; divulgar e explicar aos pacientes; estimular a representação legal de pacientes e familiares; e pedir desculpas. A utilização do DA\&O aprimora a responsabilidade médica e a segurança do paciente (Bell et al 2012). 
Em pesquisa conduzida por Ock et al (2016), com médicos e pessoas do público geral na Coreia, elencaram-se que os principais elementos para o disclosure são explicação, empatia, investigações futuras e pedidos de desculpas, além de compensação e esforços para evitar futuros erros. Evidenciou-se, ainda, que o disclosure é necessário, independentemente de seus impactos ou benefícios, sendo uma questão de moral, por parte dos profissionais, e um direito dos pacientes e familiares.

Para Adams et al (2014), além de ajudar a fortalecer a confiança do paciente no sistema de saúde, prevenir erros médicos e levar ao estabelecimento de uma cultura institucional de responsabilidade e correção de erros, a implantação do DA\&O pode levar a benefícios monetários e legais. Em revisão retrospectiva realizada pelo Sistema de Saúde da Universidade de Michigan, compararam-se as informações contidas no Banco de Dados de Gerenciamento de Risco do setor de gastroenterologia, antes e após a implantação do programa DA\&O, em 2001. O período analisado foi de 1990 a 2010. Antes da implantação, foram realizados 238.911 encontros da equipe com pacientes e familiares. Após a implantação, esse número subiu para 411.944. Apesar de a quantidade total de encontros ter aumentado, percebeu-se que a taxa de reivindicações legais por 1.000 encontros caiu de $0,16 \%$ para $0,068 \%$. O total de dólares gastos, em decorrência disso, passou de US\$ 4.743.726, na era pré-implantação, para US\$ 2.176.065, na pós-implantação, apontando uma queda de 54\% (Adams et al, 2014).

Não obstante, essa atitude não é amplamente adotada. Entre os motivos que dificultam a implantação DA\&O estão a barreira cultural, em que o médico se sente desconfortável em comunicar o erro e pedir desculpas; a barreira legal, em que há medo por parte do profissional; a barreira logística; e a barreira política. (Bell et al, 2012).

Falta de comunicação entre médico-paciente após um erro motiva os pacientes a abrirem processos, algo que poderia diminuir significativamente se os pacientes recebessem disclosures satisfatórios e adequados. De acordo com Hannawa et al (2016), as razões para essa lacuna do disclosure são múltiplas. Admitir um erro é psicologicamente difícil para os profissionais de saúde, porque fere seu orgulho profissional e obrigação de não prejudicar o paciente. Além disso, na maioria das vezes, existe uma falha em sistema de incentivo ao disclosure e falta de habilidade do médico em direcionar conversas difíceis.

McLennan et al (2015), apontam que o disclosure é umas das conversas mais complexas e difíceis na saúde, um verdadeiro desafio para médicos. Por isso, é necessário treinamento para comunicação dos erros. Entretanto, apesar do interesse de médicos em aprimorar a comunicação, a grande maioria não recebeu o treinamento adequado, mostrando uma falha dentro de hospitais e faculdades.

Em análise de conversas difíceis, a avaliação do desempenho médico e a satisfação do paciente são predominantemente associadas a habilidades não verbais por parte dos profissionais de saúde. Ou seja, os pacientes apreciam comportamentos não-verbais do seu médico durante o disclosure, o que tende a aumentar a possibilidade de perdão. (Hannawa et al, 2016). O perdão interpessoal depende de vários fatores como: diminuição do sintoma de vingança e o aumento do sentimento de benevolência, para com a pessoa que cometeu o erro. Isso pode reconstruir relações, melhorar o bem estar individual, apontar a necessidade de reconhecimento do indivíduo e trazer justiça às relações. Isso não depende apenas de sentimentos, mas também de como o paciente se sente em relação ao profissional. Os pacientes esperam um pedido de desculpas de seu médico após terem sido prejudicados por um erro, pois isso transmite um senso de responsabilidade. (Hannawa et al, 2016).

Além disso, o benefício estende-se para o profissional. McLennan et al (2015) aponta que anestesistas que já realizaram um disclosure sentiram melhora no sentimento de culpa e apresentaram maior probabilidade de concordar com a necessidade de comunicar um erro, quando comparados com médicos que não tiveram alívio ou que nunca revelaram um erro grave antes, mostrando o quanto o disclosure tem impacto emocional sobre os médicos.

Outro ponto importante no disclosure é a notificação de intercorrências entre os médicos. Em pesquisa conduzida por Alsafi et al (2011) no Al-lman Geral Hospital na Árabia Saudita com 107 médicos, abordou porque um profissional não denuncia um colega que comete um erro. 41,1\% alegaram que o motivo foi "sinto que não é minha responsabilidade", $33,7 \%$ 
concordaram com "não quero perder meu bom relacionamento com meu colega" e 16,8\% com "tenho medo de que ele/ela relate o meu erro outra vez". A respeito da não notificação de erros médicos, as quatro causas identificadas foram "nenhum incentivo à divulgação de erros", "evitar punições", "evitar danos à reputação" e "o erro não será descoberto". Apesar de 85\% concordaram com "relatórios são uma questão de ética", 66,4\% com "relatórios ajudam a aliviar sentimentos de culpa" e $83,2 \%$ com "relatório evita complicações adicionais ao paciente", 59,8\% alegam que erro deve ser mantido em sigilo e 73\% de que o problema deve ser resolvido dentro do departamento. Além disso, 60\% da amostra alegou se sentir encorajada a relatar se houvesse garantia da confidencialidade.

A pesquisa realizada por Chiang et al (2010) com 838 enfermeiras de cinco hospitais de Taiwan, apontou que $47 \%$ dos entrevistados falharam em relatar erros próprios ou de colegas de trabalho. A principal justificativa foi a cultura tradicional de profissionalismo em um ambiente que prioriza a harmonia intragrupo.

Alsafi et al (2011) destaca, ainda, que a transparência desempenha um papel importante no sucesso geral das organizações, como hospitais e comunidades médica. Para Adams et al (2014), se o profissional atua com transparência, não é necessário que exista uma relação médico-paciente prévia, admitindo abertamente a ocorrência do erro.

\subsection{Estado da arte}

Na Alemanha, "erros médicos" e "segurança do paciente" são temas cada vez mais discutidos. Isso se dá por ação da mídia, da lei de direitos dos pacientes e do Grupo de Apoio a Segurança do Paciente e a Qualidade do Cuidado, desenvolvido pela Comissão Europeia de 2013. Nesse mesmo ano, esses temas foram incluídos ao currículo estudantil (Kiesewetter et al, 2014).

Na Suíça, o disclosure tem recebido mais atenção desde que a Fundação Suíça de Segurança do Paciente foi criada em 2003. Em 2010, o Monitoramento Nacional para Gerenciamento de Riscos Clínicos, apontou que 65\% dos hospitais apresentavam uma coordenação central para gerenciamento de riscos (Mclennan et al, 2015).

Na Noruega, desde a introdução da Lei dos Serviços de Saúde Especializados da Noruega em 1999, hospitais e empresas que fornecem cuidados de saúde especializados são obrigados a relatar todos os erros médicos que resultaram, ou poderia ter resultado, em lesão do paciente para o Centro Norueguês de Conhecimento para os Serviços de Saúde. (Smeby et al, 2015)

Já na Itália, estudo realizado em 40 hospitais com 696 médicos, foi avaliado que 98,4\% dos profissionais concordam que os médicos devem discutir com colegas sobre erros médicos que ocorreram durante a clínica; 87,6\% declararam que os relatórios de erros médicos na instituição podem melhorar a qualidade cuidados para futuros pacientes. Em contrapartida, 44,5 e $44,1 \%$, respectivamente, concordaram ou não tinham certeza sobre a divulgação de erros para os pacientes. (Flotta et al, 2012).

\section{Conclusão}

Ao buscar o objetivo de analisar o uso do disclosure, como ferramenta de gestão da qualidade e segurança, realizado entre profissionais de saúde e pacientes/familiares, pode-se apontar como evidência desta revisão sistemática, que o processo de disclosure não recebe atenção suficiente na comunidade científica ou em instituições de saúde do mundo, considerando-se que poucos países apresentam práticas formalizadas e institucionalizadas por meio de protocolos ou guidelines sobre essa técnica de comunicação efetiva primordialmente humana.

Os erros acontecem e continuarão a acontecer globalmente.

É inevitável no cuidado à saúde, não obstante, os sistemas de saúde e a interação humana não estão em sintonia, portanto, importante ressaltar a hora e vez do despertar da cultura de segurança que trataria o disclosure como parte intrínseca 
de um processo de qualidade com abordagem multifacetada que tornasse a prática mais sistematizada e principalmente humanizada.

Vale pontuar a importância da educação como uma forma de promover oportunidade de aprendizado por meio dos erros, com melhorias na comunicação médico-paciente desde a graduação, promovendo uma comunicação eficaz com os pacientes e seus familiares, melhoria no trabalho interdisciplinar e uso de práticas baseadas em evidências.

A cerca de futuros trabalhos, a sugestão é a criação de questionários validados para aplicação direta a profissionais de saúde. Para que assim, órgãos públicos e privados possam criar protocolos de disclosure que atendam as necessidades de cada instituição.

\section{Referências}

Adams, M. A., Elmunzer, B. J., \& Scheiman, J. M. (2014). Effect of a health system's medical error disclosure program on gastroenterology-related claims rates and costs. The American journal of gastroenterology, 109(4), 460-464. https://doi.org/10.1038/ajg.2013.375

Alsafi, E., Bahroon, S. A., Tamim, H., Al-Jahdali, H. H., Alzahrani, S., \& Al Sayyari, A. (2011). Physicians' attitudes toward reporting medical errors-an observational study at a general hospital in Saudi Arabia. Journal of patient safety, 7(3), 144-147. https://doi.org/10.1097/PTS.0b013e31822c5a82

Bell, S. K., Smulowitz, P. B., Woodward, A. C., Mello, M. M., Duva, A. M., Boothman, R. C., \& Sands, K. (2012). Disclosure, apology, and offer programs: stakeholders' views of barriers to and strategies for broad implementation. The Milbank quarterly, 90(4), 682-705. https://doi.org/10.1111/j.14680009.2012.00679.x

Brasil (2014). Documento de referência para o Programa Nacional de Segurança do Paciente. Brasília: Ministério da Saúde.

Brasil (2015). Disclosure precisa integrar a cultura de segurança do paciente. Brasília: IBSP

Canada (2011). Canadian Disclosure Guidelines. Edmonton: Canadian Patient Safety Institute

Chiang, H. Y., Lin, S. Y., Hsu, S. C., \& Ma, S. C. (2010). Factors determining hospital nurses' failures in reporting medication errors in Taiwan. Nursing outlook, 58(1), 17-25. https://doi.org/10.1016/j.outlook.2009.06.001

Daud-Gallotti, Renata Mahfuz, Morinaga, Christian Valle, Arlindo-Rodrigues, Marcelo, Velasco, Irineu Tadeu, Martins, Milton Arruda, \& Tiberio, Iolanda Calvo. (2011). A new method for the assessment of patient safety competencies during a medical school clerkship using an objective structured clinical examination. Clinics, 66(7), 1209-1215. https://doi.org/10.1590/S1807-59322011000700015

Erdmann, Thomas Rolf, Garcia, Jorge Hamilton Soares, Loureiro, Marcos Lázaro, Monteiro, Marcelo Petruccelli, \& Brunharo, Guilherme Muriano. (2016). Profile of drug administration errors in anesthesia among anesthesiologists from Santa Catarina. Revista Brasileira de Anestesiologia, 66(1), 105-110. https://doi.org/10.1016/j.bjane.2014.06.011

Flotta, D., Rizza, P., Bianco, A., Pileggi, C., \& Pavia, M. (2012). Patient safety and medical errors: knowledge, attitudes and behavior among Italian hospital physicians. International journal for quality in health care: journal of the International Society for Quality in Health Care, 24(3), 258-265. https://doi.org/10.1093/intqhc/mzs014

Gallagher, T. H., Studdert, D., \& Levinson, W. (2007). Disclosing harmful medical errors to patients. The New England journal of medicine, 356(26), 27132719. https://doi.org/10.1056/NEJMra070568

Hannawa, A. F., Shigemoto, Y., \& Little, T. D. (2016). Medical errors: Disclosure styles, interpersonal forgiveness, and outcomes. Social science \& medicine (1982), 156, 29-38. https://doi.org/10.1016/j.socscimed.2016.03.026

Institute of Medicine (US) Committee on Quality of Health Care in America, Kohn, L. T., Corrigan, J. M., \& Donaldson, M. S. (Eds.). (2000). To Err is Human: Building a Safer Health System. National Academies Press (US).

Kalra, J., Kalra, N., \& Baniak, N. (2013). Medical error, disclosure and patient safety: A global view of quality care. Clinical Biochemistry, 46(13-14), 11611169. doi:10.1016/j.clinbiochem.2013.03.025

Kiesewetter, J., Kager, M., Lux, R., Zwissler, B., Fischer, M. R., \& Dietz, I. (2014). German undergraduate medical students' attitudes and needs regarding medical errors and patient safety--a national survey in Germany. Medical teacher, 36(6), 505-510. https://doi.org/10.3109/0142159X.2014.891008

Kisuule, F., \& Howell, E. E. (2015). Hospitalists and Their Impact on Quality, Patient Safety, and Satisfaction. Obstetrics and gynecology clinics of North America, 42(3), 433-446. https://doi.org/10.1016/j.ogc.2015.05.003

McLennan, S. R., Engel-Glatter, S., Meyer, A. H., Schwappach, D. L., Scheidegger, D. H., \& Elger, B. S. (2015). Disclosing and reporting medical errors: Cross-sectional survey of Swiss anaesthesiologists. European journal of anaesthesiology, 32(7), 471-476. https://doi.org/10.1097/EJA.0000000000000236

Motta Filho, Geraldo da Rocha, Silva, Lucia de Fatima Neves da, Ferracini, Antonio Marcos, \& Bahr, Germana Lyra. (2013). The WHO Surgical Safety Checklist: knowledge and use by Brazilian orthopedists. Revista Brasileira de Ortopedia, 48(6), 554-562. https://doi.org/10.1016/j.rboe.2013.12.010

Ock, M., Kim, H. J., Jo, M. W., \& Lee, S. I. (2016). Perceptions of the general public and physicians regarding open disclosure in Korea: a qualitative study. BMC medical ethics, 17(1), 50. https://doi.org/10.1186/s12910-016-0134-0 
Research, Society and Development, v. 10, n. 13, e67101316252, 2021

(CC BY 4.0) | ISSN 2525-3409 | DOI: http://dx.doi.org/10.33448/rsd-v10i13.16252

Raemer, D. B., Locke, S., Walzer, T. B., Gardner, R., Baer, L., \& Simon, R. (2016). Rapid Learning of Adverse Medical Event Disclosure and Apology. Journal of patient safety, 12(3), 140-147. https://doi.org/10.1097/PTS.0000000000000080

Sampaio, RF, \& Mancini, MC. (2007). Estudos de revisão sistemática: um guia para síntese criteriosa da evidência científica. Brazilian Journal of Physical Therapy, 11(1), 83-89. https://doi.org/10.1590/S1413-35552007000100013

Smeby, S. S., Johnsen, R., \& Marhaug, G. (2015). Documentation and disclosure of adverse events that led to compensated patient injury in a Norwegian university hospital. International journal for quality in health care : journal of the International Society for Quality in Health Care, 27(6), 486-491. https://doi.org/10.1093/intqhe/mzv084 